\title{
On Opening Crack Propagation in Viscoelastic Solids
}

\author{
B. N. J. Persson ${ }^{1}$
}

Received: 14 April 2021 / Accepted: 31 July 2021 / Published online: 14 August 2021

(c) The Author(s) 2021

\begin{abstract}
We show that the Persson-Brener theory of crack propagation in viscoelastic solids gives a viscoelastic fracture energy factor $G / G_{0}=1+f$ which is nearly the same as the viscoelastic factor obtained using the cohesive-zone model. We also discuss finite size effects and comment on the use of crack propagation theories for "solids" with a viscoelastic modulus that vanishes at zero frequency.
\end{abstract}

\section{Graphical Abstract}

(a) infinite slab length

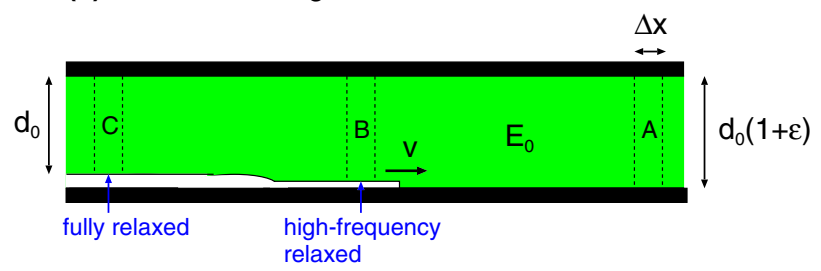

(b) finite slab length $L$

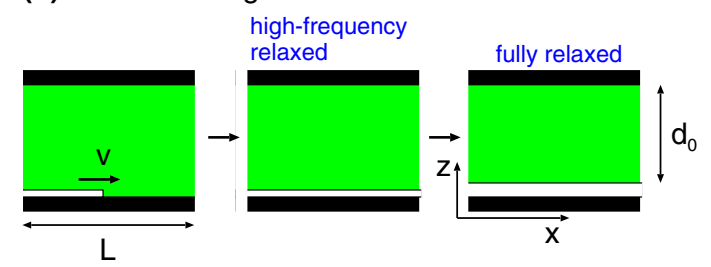

Keywords Crack · Viscoelastic $\cdot$ Finite size

\section{Introduction}

Crack propagation in viscoelastic solids, or at the interface between a viscoelastic solid and a counter surface, have many important applications, e.g., for rubber wear [1], or in adhesion and friction involving rubber-like materials [2-10]. Two different approaches have been applied to crack propagation in viscoelastic solids. One focus on the stress using the cohesive-zone model, and another is based on an energy

B. N. J. Persson

b.persson@fz-juelich.de

https://www.MultiscaleConsulting.com

1 PGI-1, FZ Jülich, Jülich, EU, Germany approach. The first approach was used by Knauss [4] and by Schapery [11, 12] and later by Hui et al [13] and by Greenwood $[14,15]$. Since the exact relation between the stress $\sigma$ and the surface separation $u$ in the cohesive (process) zone, where the bond-breaking is assumed to occur, is not known in general, in the simplest approach it was assumed that the stress is constant and equal to $\sigma_{0}$ for $0<u<h_{0}$ and $\sigma=0$ for $u>h_{0}$. The second (energy) approach was used by de Gennes [16] in a qualitative way, and by Persson and Brener [17] in a quantitative way. In the latter approach enters a cut-off radius $a_{0}$, which can be interpreted as the radius of curvature of the crack tip in the adiabatic limit, and the stress $\sigma_{\mathrm{c}}$ at the crack tip (the stress to break the adhesive or cohesive bonds). 


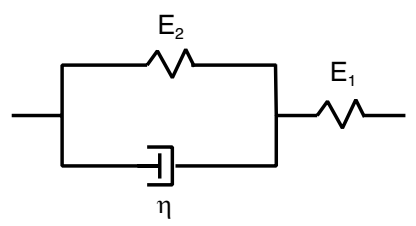

Fig. 1 Three-element viscoelastic model used in model calculation of the crack propagation energy $G(v)$. The low frequency modulus $E(0)=E_{0}=E_{1} E_{2} /\left(E_{1}+E_{2}\right)$ and the high frequency modulus $E(\infty)=E_{1}$ and the viscosity $\eta=1 / \tau$ are indicated

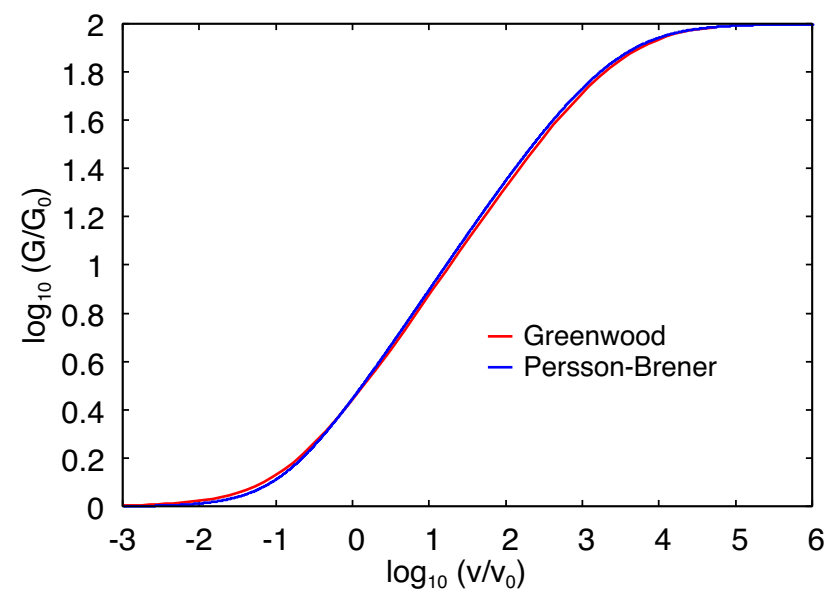

Fig. 2 The crack propagation viscoelastic factor $G / G_{0}=1+f(v)$ as a function of the crack tip speed (log-log scale) for the three level rheology model. The red curve is calculated by Greenwood using the cohesive-zone model with the adiabatic (infinitely slowly) work of adhesion $G_{0}=h_{0} \sigma_{0}$. The blue curve is from the theory of Persson and Brener assuming a crack (adiabatic) tip cut-off radius $a_{0}$. The reference velocity $v_{0}=a_{0} / \tau$ with $a_{0}=E_{0} G_{0} /\left(2 \pi \sigma_{c}^{2}\right)$ in the Persson-Brener cut-off model and $v_{0}=1.41 b / \tau$ with $b=E_{0} G_{0} / \sigma_{0}^{2}$ in the cohesive-zone model

\section{Viscoelastic Factor $\boldsymbol{G} / \mathbf{G}_{0}$}

The stress $\sigma_{0}$ in the cohesive-zone approach, is in general not the same as the stress $\sigma_{c}$ in the theory of Persson and Brener (see Appendix A). In Ref. [14] it was assumed that the stress in the process zone is a constant $\sigma=\sigma_{0}$ for $0<z<h_{0}$, where $\sigma_{0}$ can be any number as long as the (adiabatic) work of adhesion is given by $G_{0}=h_{0} \sigma_{0}$. If one use another wallwall interaction, e.g., based on the Lennard-Jones potential, one gets another relation between $G_{0}$ and $\sigma_{0}$ [15]. Since the final result depends on $\sigma_{0}$ this approach is in fact somewhat ill-defined unless the exact relation between the stress and the separation is known (which in general is not the case) and used in the theory. On the contrary in the theory of Persson and Brener only well-defined (experimental) quantities occur. Thus the adiabatic crack tip radius $a_{0}$ could in principle be measured using, e.g., an electron microscope.
In any case for cohesive cracks we expect $a_{0}$ to be of order the length of the polymer chains between the cross links, i.e., typically of order $1 \mathrm{~nm}$.

In Ref. [17, 18], it was stated that the Persson-Brener theory gives nearly the same result for the viscoelastic factor $G / G_{0}=1+f(v)$ as the cohesive-zone model. Thus the numerical results for $G / G_{0}$ obtained by Greenwood and by Hui et al for the three-element viscoelastic model (see Fig. 1) is nearly the same as predicted by the Persson-Brener theory if one chooses $\sigma_{0}$ to get the best possible overlap between the two curves (which means using $\sigma_{0} \approx 3 \sigma_{c}$ ), see Fig. 2 (see Appendix B for the equations used in the calculations). Shifting like this is the only meaningful way to compare the factor $G / G_{0}$ between the two theories, because the velocity normalization factor in the cohesive-zone approach depends on the cut-off stress $\sigma_{0}$ which differ from $\sigma_{\mathrm{c}}$ (see Appendix A).

The fact that the two curves in Fig. 2 agree so well indicate that the viscoelastic factor $G / G_{0}$ is not sensitive to the form of the bond-breaking process at the crack tip. This is also consistent with the observation that using a LennardJones type of wall-wall interaction potential [15], or another shorter range potential [19], result in nearly the same result as presented above.

(a) infinite slab length

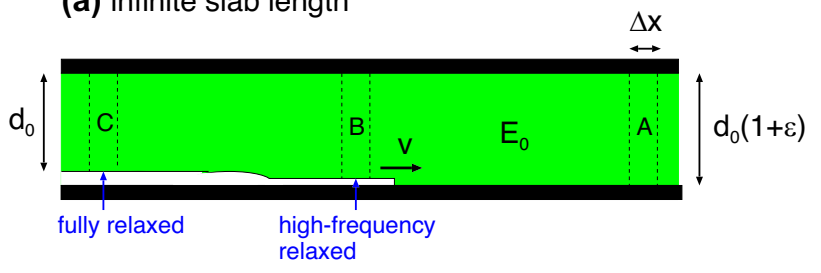

(b) finite slab length $\mathrm{L}$

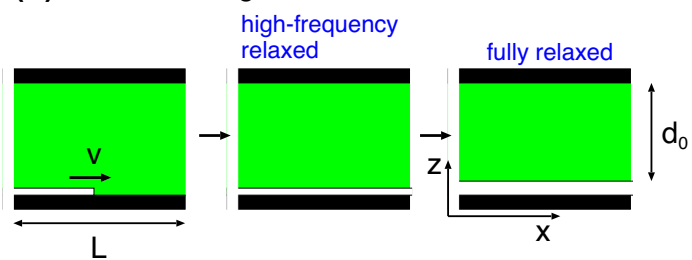

Fig. 3 Crack propagation in a viscoelastic slab clamped between two rigid flat surfaces. a The slab is infinite long $(L=\infty)$ in the crack propagation direction ( $x$-direction). b The slab is of finite length $L$. In a the segment (of width $\Delta x$ ) at $\mathrm{A}$ is stretched with the strain $\epsilon$. At $\mathrm{B}$, the strain is reduced (high frequency relaxation) by $\Delta \epsilon=\sigma_{0} / E_{1}$ due to the abrupt decrease in the tensile stress from $\sigma_{0}$ to 0 when the crack tip pass the segment. At $\mathrm{C}$, far away from the crack tip, the strain vanish due to (slow) viscoelastic relaxation 


\section{Finite Size Effect}

At high crack tip speed (or at low temperatures) the main contribution to the viscoelastic energy dissipation comes from a region far from the crack tip. This follows from dimensional arguments: the perturbing deformation frequencies from the moving crack (velocity $v$ ) a distance $r$ away from the crack tip must be of order $v / r$. Thus close to the crack tip the rubber will effectively be in the glassy state (elastic response) and the dominant contribution to the energy dissipation will come from regions far from the crack tip. Hence if the solid has a finite extent, say of linear dimension $L$, at high enough crack tip speed the solid will, from the point of view of viscoelastic dissipation, effectively be in the glassy elastic state everywhere and no viscoelastic energy dissipation will occur during the crack propagation. Thus one may be tempted to claim that for finite solids $f \rightarrow 0$ as $v \rightarrow \infty$. This result was used by de Gennes to argue that for the slab geometry (thickness $d_{0}$ ), as occur for example in pressure sensitive adhesives, for large crack tip speed the crack propagation energy $G(v)$ will decrease with increasing $v$ which may result in mechanical instabilities [16]. However, I will now shown in that this argument is in fact not correct and $G(v)$ does not decrease for large velocities (see also Ref. [20]).

Figure 3a shows a fast moving opening crack in a thin viscoelastic slab (thickness $d_{0}$ ) under tension. The slab is assumed to be infinite long in the crack propagation direction. The slab is elongated by $d_{0} \epsilon_{0}$, and we wait until a fully relaxed state is formed before inserting the crack. Thus the elastic energy stored in the strip A of width $\Delta x$ and volume $\Delta V=w d_{0} \Delta x$ (where $w$ is the width of the solid in the $y$-direction) is

$U_{0}=\frac{1}{2} \sigma_{0} \epsilon_{0} \Delta V=\frac{\sigma_{0}^{2}}{2 E_{0}} \Delta V$.

This energy is partly used to break the interfacial bonds and partly dissipated due to the material viscoelasticity. The crack propagation energy $G=U_{0} /(w \Delta x)=\sigma_{0}^{2} d_{0} /\left(2 E_{0}\right)$.

Consider now the slab $\Delta x$ as it moves from one side of the crack to the other side. During this transition, it will experience an (elongation) stress $\sigma(t)$ which for a very fast moving crack can be considered as a step function where $\sigma=\sigma_{0}$ for $t<0$ and $\sigma=0$ for $t>0$, where $t=0$ correspond to the case where the segment $\Delta x$ is at the crack tip. The viscoelastic material will respond to this steplike change in the stress with its high frequency modulus $E_{1}$ so the strain in the segment $\Delta x$ will abruptly drop by $\Delta \epsilon=\sigma_{0} / E_{1}$ as the crack pass the segment. The drop in the elastic energy
$\Delta U=\frac{1}{2} \sigma_{0} \Delta \epsilon \Delta V=\frac{\sigma_{0}^{2}}{2 E_{1}} \Delta V$

is used to break the interfacial bonds (energy $\Delta \gamma=G_{0}$ per unit surface area), i.e. $G_{0}=\Delta U /(w \Delta x)$ or

$G_{0}=\frac{\sigma_{0}^{2}}{2 E_{1}} d_{0}=\frac{\sigma_{0}^{2} d_{0}}{2 E_{0}} \frac{E_{0}}{E_{1}}=G \frac{E_{0}}{E_{1}}$,

so that $G=G_{0} E_{1} / E_{0}$. The remaining elastic energy stored in the segment $\Delta x$,

$U=U_{0}-\Delta U=\frac{1}{2} \sigma_{0}^{2}\left(\frac{1}{E_{0}}-\frac{1}{E_{1}}\right) \Delta V$,

is dissipated in the slow viscoelastic relaxation occurring far away from the crack tip so that finally the material reach its fully relaxed state (zero strain and stress).

For a solid with a finite extent in all directions the fast relaxation process at the crack tip is the same as above so the result $G=G_{0} E_{1} / E_{0}$ still holds. However for a fast moving crack the time, it takes for the crack to fracture the whole interface, $\Delta t=L / v$ is so short that in accordance with the discussion presented earlier, negligible viscous energy relaxation has occurred during the crack propagation act. Thus when the crack separate the two solids the viscoelastic solid is still in a strained state. Only after a possible long time period it will return to a strain- (and stress) free state. Thus in this case, the viscoelastic energy dissipation occur in a process separated from the actual crack propagation (see Fig. $3 b$ ), and this fact was overlooked in the earlier energy-based discussions of finite size effects [21-23]. However, this argument does not exclude that the $G(v)$ relation depends on the system size for intermediate crack tip velocities.

\section{Loading Followed by Immediate Unloading}

The discussions of finite size effects presented above has assumed that at the onset of pull-off, the viscoelastic solid far from the crack tip is in a fully relaxed state characterized by the low frequency modulus $E_{0}$. Only in this case will the crack propagation energy $G \rightarrow G_{0} E_{1} / E_{0}$ as the crack tip velocity $v \rightarrow \infty$. Consider, for example, moving a rigid sphere in and out of contact with a viscoelastic halfspace with the speed $\pm v$. After bringing the sphere in contact with the viscoelastic solid, the contact is kept fixed for a time period $t_{0}$ before the pull-off. If $t_{0}$ is long enough (e.g. $t_{0} \gg>\tau$ for the three-element rheology model) the deformation field resulting from the loading (indentation) can, before the onset of pull-off, relax to its fully relaxed 


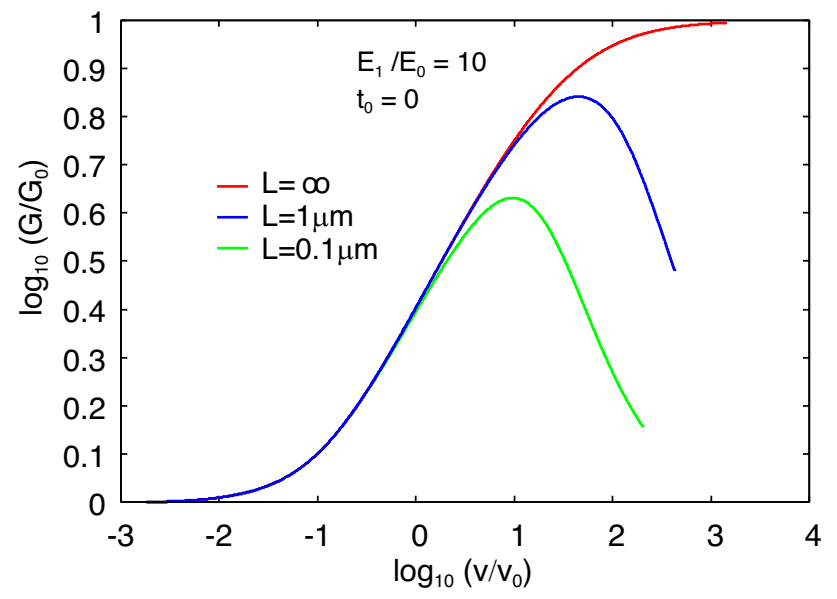

Fig. 4 The crack propagation energy factor $G / G_{0}=1+f(v)$ as a function of the crack tip speed (log-log scale) for the three level rheology model with $E_{1} / E_{0}=10$ and $\tau=1 \mathrm{~s}$. We have assumed that pull-off occur immediately after the loading, involving the same approach and pull-off speeds $\pm v$. The red curve is calculated using the theory of Persson and Brener assuming a crack (adiabatic) tip cut-off radius $a_{0}=1 \mathrm{~nm}$. The blue and green curves are the results obtained using the theory presented in Ref. [21] for the (finite) size parameter $L=1 \mu \mathrm{m}$ and $L=0.1 \mu \mathrm{m}$, respectively. The reference velocity $v_{0}=a_{0} / \tau$

state, characterized by the low frequency modulus $E_{0}$. In this case, the discussion presented in Sec. 3 is valid, and if the pull-off speed is high, an indentation is left on the surface of the viscoelastic solid which only slowly relax (on the time-scale $\sim \tau$ ) to its undeformed state.

Assume now $t_{0}=0$. In this case the perturbing deformation frequencies, in the region of the viscoelastic solid which undergoes deformations, will be of order $v / r_{0}$ or higher, where $r_{0}$ is the maximum radius of the contact region between the sphere and the halfspace. For high enough speed $v$ these frequencies will be in the glassy frequency region, and the viscoelastic solid will during the whole loading-unloading respond as an elastic solid with the high frequency modulus $E_{1}$. Thus in this case the crack propagation energy $G \rightarrow G_{0}$ as $v \rightarrow \infty$. On the other hand for low enough speed $v$ the solid will be in the rubbery region (characterized by the low frequency modulus $E_{0}$ ) everywhere except very close to the crack tip where the perturbing frequencies $v / r$ (where $r$ is the distance from the crack tip) are high. Hence even when $t_{0}=0$ for low enough pull-off speed, the viscoelastic crack propagation theory presented in Sec. 3 is valid. It follows that the effective crack propagation energy will have the general form shown by the blue (or green) line in Fig. 4 .

The blue (and green) line in Fig. 4 was obtained using the theory developed in Ref. [21]. This theory gives the effective crack propagation energy during pull-off (opening crack propagation) assuming that in the absence of adhesion, there is no elastic energy left in the viscoelastic solid after removing the spherical indenter. In reality, even if $t_{0}=0$, some elastic deformation energy will be left after unloading. However, there is a qualitatively difference between long contact time and short contact time. First note that all real rubber materials have relaxation times which extend over more than 10 decades in time. When the sphere is in contact with a viscoelastic solid (rubber) for some (long) time period $t_{0}$ the relaxation modes in the rubber with relaxation times $\tau<t_{0}$ will all be activated (displaced) and the time needed for the indentation to relax back to the undeformed state will be of order $t_{0}$ since the displacement of a mode with the relaxation time $\tau$ will, after removing the contact, decay as $\sim \exp (-t / \tau)$ with increasing time. Thus, all the activated relaxation modes, with relaxation times longer than the retraction time period, will remain activated (i.e., in the displaced state) at the end of the pull-off. However, when retraction follows the approach without a delay $\left(t_{0}=0\right)$ the rubber relaxation modes activated during approach will to some extent be able to relax during the retraction. Of course not fully relaxed, but still there is a difference from the first case where $t_{0}$ is large compared to the indentation and retraction time periods. This argument is qualitative, but could be made more accurate.

The $G(v)$ curve the theory predict (blue and green lines in Fig. 4) [21] is nearly the same as obtained in a recent study by Afferrante and Violano [24] using a finite element model with Lennard-Jones interaction forces between a rigid sphere and a viscoelastic solid with a flat surface. Note that in this case there will be a finite size effect, as indeed observed in Ref. [24]. Thus, for a very large sphere the (maximum) radius of the contact region, $r_{0}$, is very large, and the deformation frequencies $v / r_{0}$ will, for all velocities of interest, be so low that the viscoelastic solid will respond as a soft elastic solid (rubbery region) everywhere, except very close to the crack tip. In this case $G \approx G_{0} E_{1} / E_{0}$ for high (but not too high) velocities, as indicated by the red curve in Fig. 4.

Recent numerical simulation studies [19, 24] have shown that, even when we start with the fully relaxed state, the work $W$ to pull-off a rigid sphere or cylinder from a viscoelastic halfspace decreases with increasing pull-off speed $v_{\mathrm{p}}$ for large $v_{\mathrm{p}}$. Thus the $W\left(v_{\mathrm{p}}\right)$ curve looks on a log-log scale roughly like a Gaussian. This result appears at first surprising since the crack propagation energy $G(v)$ increases monotonically with the crack tip speed $v$. Hence, if the work would be given by the area $A_{0}$ which is broken (which in the simulations was the same independent of the pull-off speed as the simulations always started from the fully relaxed state at vanishing applied force) times the crack propagation energy, then $W$ should increase with increasing $v_{\mathrm{p}}$. In particular, for very large pull-off velocity $W\left(v_{\mathrm{p}}\right)$ should be equal to $A_{0} G_{0}\left(E_{1} / E_{0}\right)$. However, as the pull-off speed 
increases, the snap-off occur for larger contact width (given by $w=2\left(2 G(v) R^{2} / \pi E^{*}\right)^{1 / 3}$ for the cylinder case) and if we assume that during snap-off the solid deform so quickly that the viscoelastic crack propagation theory is not valid, and that the effective $G \approx G_{0}$ rather than $G \approx G_{0}\left(E_{1} / E_{0}\right)$, then this would explain why the work to separate the solids decreases with increasing $v_{\mathrm{p}}$ for large pull-off speed.

Let us briefly discuss another application of the theory above. When a rubber block slides on a hard and rough substrate surface, such as an asphalt road surface, the rubber road contact will in general not be complete, but it will consist of many small asperity contact regions, and the area of real contact is usually a very small fraction of the nominal contact area. A very important contribution to the friction force is derived from the interaction between the rubber molecules and the road surface in the area of real contact. For clean surfaces two different (adhesive) contributions to the frictional force have been considered, namely from the opening crack on the exit side of the asperity contact region, and from a bonding-stretching-debonding process within the area of real contact. The crack propagation is very similar to the sphere approach-retraction cycle without a waiting time period (i.e. $t_{0}=0$ ), and since the asperity contact regions in general are very small and the sliding speed is relatively high, it follows that the crack propagation energy $G(v)$ may be only slightly increased by viscoelasticity rather than the large enhancement expected when the strain field is fully relaxed far from the crack tip.

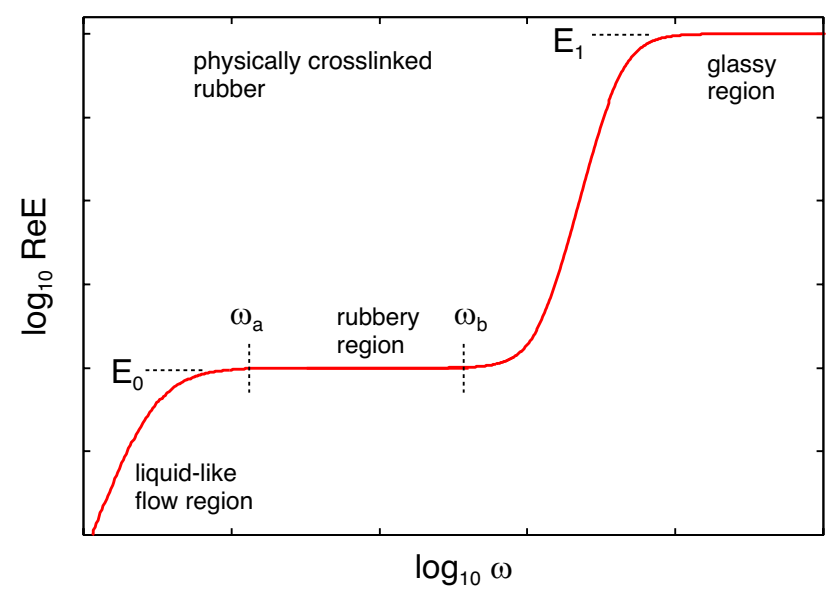

Fig. 5 The real part of the viscoelastic modulus as a function of frequency (log-log scale) for a physically cross linked polymer, which behaves as a fluid for small frequencies, corresponding to long times (schematic). The modulus $E_{0}$ in the rubbery region $\omega_{\mathrm{a}}<\omega<\omega_{\mathrm{b}}$ is approximately constant and much smaller than the modulus $E_{1}$ in the glassy region (high frequencies)

\section{Application to "Solids" with $E(\omega=0)=0$}

In one application Schapery studied viscoelastic crack propagation in "solids" which has a viscoelastic modulus that vanish for zero frequency (i.e., a relaxation modulus which vanish for long times) $[11,12]$. Such a "solid" is really a liquid with a non-Newtonian and possible complex rheology, and in this case, no rigorous crack propagation theory can be developed. Thus, for example, the Johnson, Kendall, and Roberts (JKR) adhesion theory [25], can be applied also to viscoelastic solids if the deformations far from the crack tip (here the line separating the contact area from the noncontact area), is characterized by the low frequency (fully relaxed state) elastic modulus $E_{0}=E(\omega=0)$, which would vanish in the present case. We note, however, that physically cross linked polymer materials may have a rubbery plateau (see Fig. 5 and Ref. [26, 27]) for $\omega_{\mathrm{a}}<\omega<\omega_{\mathrm{b}}$, and behave as an elastic solid for all practical time scales $1 / \omega_{\mathrm{b}}<t<1 / \omega_{\mathrm{a}}$, in which case the cohesive-zone (or Persson-Brener) theory could still be applied but with $E_{0}$ being the modulus in the rubbery plateau region. I note that the Schapery theory is general, and can be applied to solids with arbitrary viscoelastic modulus.

If the adiabatic crack tip radius $a_{0}$ is treated as a constant as the low frequency modulus $E_{0}$ is varied then the viscoelastic factor $G / G_{0}$ is independent of $E_{0}$ for low enough velocities and hence well-defined even in the limit $E_{0}=0$ (see Appendix C). Nevertheless, $a_{0}$ is not well-defined in the limit $E_{0}=0$ since it refer to the adiabatic limit where the "solid" responds as a fluid, where no crack-like defects can occur.

\section{Discussion}

In a recent series of papers, Ciavarella et al [28, 29] have criticized the Persson-Brener theory and claimed that the PerssonBrener theory [17] gives a viscoelastic factor $G / G_{0}=1+f$ which differ strongly from what Ciavarella et al denote as the exact viscoelastic factor obtained using the cohesive-zone model $[13,14]$. We have shown above that both theories gives nearly the same result for $G / G_{0}$ if the quantity $\sigma_{0}$ in the cohesive-zone theory is chosen appropriately!

In another paper Popov [30] claim that viscoelasticity increases the JKR pull-off force with a factor $E_{1} / E_{0}$ in the quasi-static limit. This result is incorrect: in the quasi-static case, the work of adhesion is not influenced by the viscoelasticity and only in the limit $v \rightarrow \infty$ is the work of adhesion increased by the factor of $E_{1} / E_{0}$. The result of Popov would hold if there would be no shortest length scale in the problem but in reality there is a short distance cut-off denoted by $a_{0}$ above. 


\section{Appendix A}

In the Persson-Brener approach, there is no cohesive-zone so the cut-off stress $\sigma_{\mathrm{c}}$ is not exactly the same as the cut-off stress $\sigma_{0}$ in the cohesive-zone model (now they differ by a factor of $\sim 3$ ). In fact, the cut-off stress in the cohesive-zone model depends on the form of the wall-wall interaction stress (assuming the work of adhesion is given) which is not known in general, and may be very complex when processes such as chain pull-out occur.

One can derive a relation between $\sigma_{c}$ and $\sigma_{0}$ by demanding that the stress field away from the process zone takes the same form $K(2 \pi r)^{-1 / 2}$ in both cases. For the general case where $\sigma_{0}$ is not a constant, but varies with the surface separation, in the cohesive-zone model

$\frac{K}{(2 \pi)^{1 / 2}}=\frac{1}{\pi} \int_{0}^{\infty} d x^{\prime} \sigma_{0}\left(x^{\prime}\right)\left(x^{\prime}\right)^{-1 / 2}$

In the Persson-Brener theory

$\sigma_{c}=\frac{K}{(2 \pi a)^{1 / 2}}$

Using (A1) and (A2) gives

$\sigma_{c}=\frac{1}{\pi} \int_{0}^{\infty} d x^{\prime} \sigma_{0}\left(x^{\prime}\right)\left(a x^{\prime}\right)^{-1 / 2}$

In the simplest case where $\sigma_{0}(x)$ is a constant $\sigma_{0}$ for $0<z<h_{0}$ and $0<x<l$ and zero otherwise we get

$\sigma_{c}=\frac{2}{\pi}\left(\frac{l}{a}\right)^{1 / 2} \sigma_{0}$

The best way to obtain the cut-off radius $a_{0}$ (for low crack tip velocity) in the Persson-Brener model is to study the crack tip directly and fit it to a circular segment with radius $a_{0}$. This is easy to do in numerical simulations like in Ref. [19]. In real experimental situations it may be harder (because $a_{0}$ is very small) but if one can measure $a(v)$ for large crack tip speed, where the radius is enhanced by a factor of $E_{1} / E_{0}$, one can calculate $a_{0}$ from $a_{0}=\left(E_{0} / E_{1}\right) a(\infty)$.

\section{Appendix B}

Here we give the equations for viscoelastic crack propagation used in the calculations in Fig. 2.

When a strip of a viscoelastic material is exposed to an oscillating strain $\epsilon(\omega) \exp (-i \omega t)$ the amplitude of the oscillating stress

$\sigma(\omega)=E(\omega) \epsilon(\omega)$

This equation define the viscoelastic modulus $E(\omega)$, which is a complex quantity, where the imaginary part is related to energy dissipation (transfer of mechanical energy into the disordered heat motion). In the calculation in Fig. 2, we used the three-element rheological model illustrated in Fig. 1. For this model the viscoelastic modulus

$E=\frac{E_{0} E_{1}(1-i \omega \tau)}{E_{1}-i \omega \tau E_{0}}$

The ratio between the high frequency and low frequency modulus, $E_{1} / E_{0}$, is typically very large, e.g., $\sim 1000$.

In the Persson-Brener theory, the crack propagation energy $G(v)=G_{0} a(v) / a_{0}$, where $a(v)$ is the velocity-dependent effective crack tip radius, and where $a_{0}=E_{0} G_{0} /\left(2 \pi \sigma_{\mathrm{c}}^{2}\right)$. The fracture energy $G(v)$ satisfy [17]

$\frac{G_{0}}{G}=1-E_{0} \frac{2}{\pi} \int_{0}^{\omega_{c}} d \omega F(\omega) \frac{1}{\omega} \operatorname{Im} \frac{1}{E(\omega)}$

where

$F(\omega)=\left[1-\left(\frac{\omega}{\omega_{c}}\right)^{2}\right]^{1 / 2}$

where $\omega_{c}=2 \pi v / a$. For numerical calculations it is more convenient to reformulate (B2) into $[7,21]$

$\frac{G_{0}}{G}=1-\frac{E_{1} \frac{2}{\pi} \int_{0}^{\omega_{c}} d \omega \frac{1}{\omega} F(\omega) \operatorname{Im} \frac{1}{E(\omega)}}{1+E_{1} \frac{2}{\pi} \int_{0}^{\infty} d \omega \frac{1}{\omega} \operatorname{Im} \frac{1}{E(\omega)}}$.

Since $\omega_{c}$ depends on $a$ (and hence on $G$ since $G=G_{0} a / a_{0}$ ) this is an implicit equation for $G(v)$. Thus the theory gives both crack propagation energy $G(v)$ and the (velocitydependent) radius of the crack tip,

$a(v)=a_{0} \frac{G(v)}{G_{0}}=\frac{E_{0} G}{2 \pi \sigma_{c}^{2}}$.

Since $E(\omega)$ typically varies with $\omega$ over very many decades in frequency, for the numerical evaluation of the integrals in (B3) it is convenient to write (see Ref. [7]) $\omega=\omega_{0} e^{x}$, so that if $\omega$ varies over $\sim 30$ decades, $x$ varies only by a factor $\sim 100$.

In the cohesive-zone model assuming that the stress is constant and equal to $\sigma_{0}$ for $0<u<h_{0}$ and $\sigma=0$ for $u>h_{0}$, and assuming the viscoelastic modulus (B1), one get [14]:

$\frac{G_{0}}{G}=\frac{E_{0}}{E_{1}}+\frac{1}{2}\left(1-\frac{E_{0}}{E_{1}}\right) \alpha \int_{0}^{1} d x H(x) e^{-\alpha(1-x)}$,

where $\alpha=l /(v \tau)$ and

$H(x)=2 x^{1 / 2}-(1-x) \ln \left(\frac{1+x^{1 / 2}}{1-x^{1 / 2}}\right)$,

and the width $l$ of the crack tip process zone 


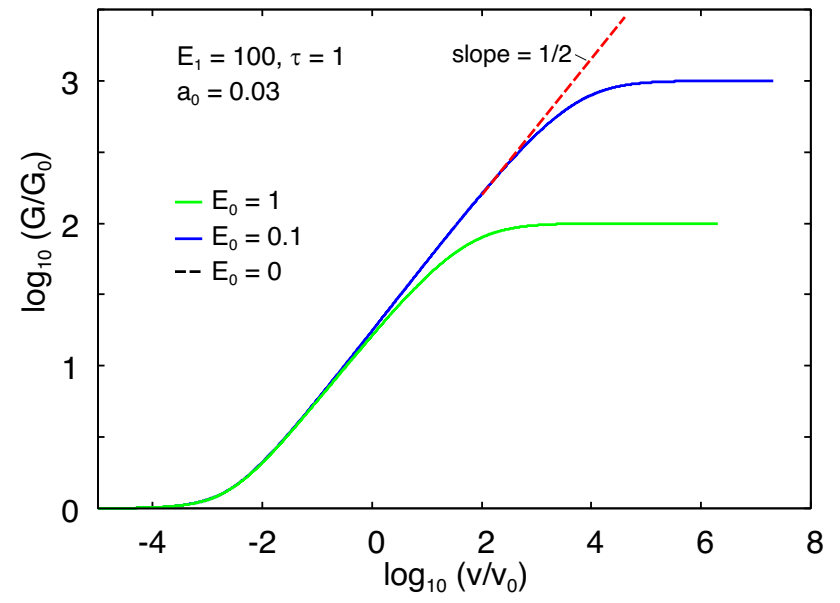

Fig. 6 The viscoelastic factor $G / G_{0}$ as a function of the crack tip speed (log-log scale) for the case $E_{1}=100, \tau=1$ and $E_{0}=1$ (green curve), $E_{0}=0.1$ (blue curve) and $E_{0}=0$ (red dashed curve). In the calculation, we have used the adiabatic crack tip radius $a_{0}=0.03$. The reference velocity $v_{0}=a_{0} / \tau$

$l=\frac{\pi}{4} \frac{E_{0} G}{\sigma_{0}^{2}}$

Here we have assumed plane stress; for plane strain $E$ must be replaced with $E /\left(1-v^{2}\right)$, where $v$ is the Poisson ratio. Since $l$ depends on $G$, (B5) is an implicit equation for $G(v)$.

\section{Appendix C}

Using the viscoelastic modulus (B1), which we can also write as

$\frac{1}{E}=\frac{1}{E_{1}}+\left(\frac{1}{E_{0}}-\frac{1}{E_{1}}\right) \frac{1}{1-i \omega \tau}$,

in (B2) and denoting $\omega=\omega_{c} x$ gives

$\frac{G_{0}}{G}=1-\left(1-\frac{E_{0}}{E_{1}}\right) \frac{2}{\pi} \int_{0}^{1} d x\left(1-x^{2}\right)^{1 / 2} \frac{\xi}{1+\xi^{2} x^{2}}$,

where $\xi=2 \pi v \tau / a=2 \pi\left[v \tau / a_{0}\right] G_{0} / G$. Hence $G / G_{0}$ depends only on $v \tau / a_{0}$ and on $E_{0} / E_{1}$ and is well-defined also in the fluid limit $E_{0}=0$.

Fig. 6 shows the viscoelastic factor $G / G_{0}$ as a function of the crack tip speed on a log-log scale. We have used $E_{1}=100, \tau=1$ and $E_{0}=1$ (green curve), $E_{0}=0.1$ (blue curve) and $E_{0}=0$ (red dashed curve), and the adiabatic crack tip radius $a_{0}=0.03$. Note that for low crack tip speed $G / G_{0}$ is independent of $E_{0}$. In fact, for $E_{0}=0$ the curve extend linearly (on the log-log scale) towards infinite $v$ with the slope $1 / 2$ corresponding to $G \sim v^{1 / 2}$ (dashed line in the figure). For a more realistic viscoelastic modulus the exponent $\alpha$ in $G \sim v^{\alpha}$ will be different from $1 / 2$ as discussed elsewhere [17].

Acknowledgements I thank J.A. Greenwood for supplying the numerical data used for the $G(v) / G_{0}$ curve for the cohesive-zone model (the red curve in Fig. 1). I thank G. Carbone and M. Müser for comments on the manuscript.

Funding Open Access funding enabled and organized by Projekt DEAL.

\section{Declarations}

Conflict of interest The authors declare that they have no conflict of interest.

Open Access This article is licensed under a Creative Commons Attribution 4.0 International License, which permits use, sharing, adaptation, distribution and reproduction in any medium or format, as long as you give appropriate credit to the original author(s) and the source, provide a link to the Creative Commons licence, and indicate if changes were made. The images or other third party material in this article are included in the article's Creative Commons licence, unless indicated otherwise in a credit line to the material. If material is not included in the article's Creative Commons licence and your intended use is not permitted by statutory regulation or exceeds the permitted use, you will need to obtain permission directly from the copyright holder. To view a copy of this licence, visit http://creativecommons.org/licenses/by/4.0/.

\section{References}

1. Persson, B.N.J.: Theory of powdery rubber wear. J. Phys.: Condens. Matter 21, 485001 (2009)

2. Tiwari, A., Dorogin, L., Tahir, M., Stöckelhuber, K.W., Heinrich, G., Espallargas, N., Persson, B.N.J.: Rubber contact mechanics: adhesion, friction and leakage of seals. Soft Matter 13, 9103 (2017)

3. Tiwari, A., Dorogin, L., Bennett, A.I., Schulze, K.D., Sawyer, W.G., Tahir, M., Heinrich, G., Persson, B.N.J.: The effect of surface roughness and viscoelasticity on rubber adhesion. Soft matter 13, 3602 (2017)

4. Knauss, W.G.: A review of fracture in viscoelastic materials. Int. J. Fract. 196, 99 (2015)

5. Creton, C., Ciccotti, M.: Fracture and adhesion of soft materials: a review. Rep. Progress Phys. 79, 046601 (2016)

6. Slootman, J., Waltz, V., Yeh, C.J., Baumann, C., Göstl, R., Comtet, J., Creton, C.: Quantifying rate-and temperature-dependent molecular damage in elastomer fracture. Phys. Rev. X 10, 041045 (2020)

7. Persson, B.N.J., Albohr, O., Heinrich, G., Ueba, H.: Crack propagation in rubber-like materials. J. Phys.: Condens. Matter 17, R1071 (2005)

8. Gent, A.N.: Adhesion and strength of viscoelastic solids. Is there a relationship between adhesion and bulk properties? Langmuir 12, 4492 (1996)

9. Carbone, G., Persson, B.N.J.: Hot cracks in rubber: origin of the giant toughness of rubberlike materials. Phys. Rev. Lett. 95, 114301 (2005)

10. Carbone, G., Persson, B.N.J.: Crack motion in viscoelastic solids: the role of the flash temperature. Eur. Phys. J. E 17, 261 (2005) 
11. Schapery, R.A.: A theory of crack initiation and growth in viscoelastic media, Int. J. Fract. 11, (Part I) 141 (1975)

12. Schapery, R.A.: A theory of crack initiation and growth in viscoelastic media II. Approximate methods of analysis. Int. J. Fract. 11, 369 (1975)

13. Hui, C.Y., Xu, D.B., Kramer, E.J.: A fracture model for a weak interface in a viscoelastic material (small scale yielding analysis). J. Appl. Phy. 72, 3294 (1992)

14. Greenwood, J.A.: The theory of viscoelastic crack propagation and healing. J. Phys. D: Appl. Phys. 37, 2557 (2004)

15. Greenwood, J.A.: Viscoelastic crack propagation and closing with Lennard-Jones surface forces. J. Phys. D: Appl. Phys. 40, 1769 (2007)

16. de Gennes, P.G.: Soft adhesives. Langmuir 12, 4497 (1996)

17. Persson, B.N.J., Brener, E.A.: Crack propagation in viscoelastic solids. Phys. Rev. E 71, 036123 (2005)

18. Rodriguez, N., Mangiagalli, P., Persson, B.N.J.: Viscoelastic crack propagation: review of theories and applications, In: Heinrich G., Kipscholl R., Stocek R. (eds) Fatigue Crack Growth in Rubber Materials. Advances in Polymer Science, vol 286. (2020). Springer, Cham. https://doi.org/10.1007/12_2020_76

19. Müser, M.H., Persson, B.N.J.: Crack and pull-off dynamics of adhesive, viscoelastic solids, arXiv:2108.02031

20. Persson, B.N.J.: A simple model for viscoelastic crack propagation. Eur. Phys. J. E 44. Article number 3,(2021)

21. Persson, B.N.J.: Crack propagation in finite-sized viscoelastic solids with application to adhesion. EPL 119, 18002 (2017)

22. Saulnier, F., Ondarcuhu, T., Aradian, A., Raphae11, E.. : Adhesion between a viscoelastic material and a solid surface. Macromolecules 37, 1067 (2004)

23. Ciavarella, M., Papangelo, A.. : Effects of finite thickness on crack propagation in viscoelastic materials. Eng. Fract. Mech. 248, 107703 (2021)
24. Afferrante, L., Violano, G.: On the effective surface energy in viscoelastic Hertzian contacts, arXiv:submit/3829951

25. Johnson, K.L., Kendall, K., Roberts, A.D.: Surface energy and the contact of elastic solids. Proc. Royal Soc London. A. Math. Phys. Sci. 324, 301 (1971)

26. Saphiannikova, M., Toshchevikov, V., Gazuz, I., Petry, F., Westermann, S., Heinrich, G.: Multiscale approach to dynamic-mechanical analysis of unfilled rubbers. Macromoleculrs 47, 4813 (2014)

27. Tanaka, F., Edwards, S.F.: Viscoelastic properties of physically cross-linked networks. Transient Network Theory, Macromolecules 25, 1516 (1992)

28. Ciavarella, M.: A comparison of Gent-Schultz law with cohesive models of viscoelastic subcritical crack propagation, Prepring (2021), see: https://www.researchgate.net/publication/350325255 _A_comparison_of_Gent-Schultz_law_with_cohesive_models_ of_viscoelastic_subcritical_crack_propagation

29. Ciavarella, M., Mcmeeking, R.: Exact solutions to the viscoelastic crack problem with power law creep compliance materials, and comparison with the Schapery and Persson-Brener approximate solutions, Prepring (2021), see: https://www.researchgate. net/publication/350431355_Exact_solutions_to_the_viscoelast ic_crack_problem _with_power_law_creep_compliance_mater ials_and_comparison_with_the_Schapery_and_Persson-Brener _approximate_solutions

30. Popov, V.L.: Energetic criterion for adhesion in viscoelastic contacts with non-entropic surface interaction. Rep. Mech. Eng. 2, 57 (2021)

Publisher's Note Springer Nature remains neutral with regard to jurisdictional claims in published maps and institutional affiliations 